\title{
CONCERNING UNIQUENESS-BASES OF FINITE GROUPS WITH APPLICATIONS TO $p$-GROUPS OF CLASS $2^{*}$
}

\author{
BY \\ CHARLES HOPKINS $\dagger$
}

A uniqueness-basis ( $U$-basis) of a finite group $G$ has been defined as "an ordered set of elements $Q_{1}, Q_{2}, \cdots, Q_{\rho}$ such that every element of $G$ can be expressed uniquely in the form $Q_{1}{ }^{x_{1}} Q_{2}{ }^{x_{2}} \cdots Q_{\rho}{ }^{x_{\rho}}$, where each $x_{i}$ is a least positive residue modulo the order of $Q_{i} . " \ddagger$ In the case of the abelian groups the notion of $U$-basis is of undisputed importance: the theorem that every finite abelian group $A$ has a $U$-basis may fairly be regarded as the cornerstone of the theory of abelian groups.

In the case of most non-abelian groups, however, the concept of $U$-basis is of doubtful advantage, especially in the general form above. Of greater usefulness, naturally, would be a "simplest type" of $U$-basis for the group under consideration. But the problem of constructing a definition of a "normal form" which shall be significant for reasonably general categories-the non-abelian $p$-groups, say-is an exceedingly difficult one. We offer a tentative definition in the case of the regular $p$-groups $\S(\$ \$ 2-3)$, which have, in common with the abelian $p$-groups, the property that the orders of the elements in every $U$-basis constitute a set of invariants of the group. In $\$ 4$ we shall show how a "normal" $U$-basis may be used in constructing for every regular $p$-group $G$ of class 2 a simply-isomorphic representation by $l$-matrices $\S$ -matrices whose coordinates are residue classes modulo certain powers of $p$. These representations of $G$ are of interest in that they usually involve a much smaller number of rows than do the matrix-representations whose coordinates are in a field. (The $l$-matrices are by no means novel; they have long been used for representing automorphisms of abelian p-groups.) In $\$ 5$ we shall discuss the representation by $l$-matrices of the group of isomorphisms of $G$, and in $\$ \S 6-7$ we shall describe, very briefly, a representation of $G$ as a multiplicative group in a finite ring.

1. In this section we shall state-for the most part without proof-several theorems which afford a set of criteria for the existence of a $U$-basis in a finite group $G$.

* Presented to the Society, September 5, 1936; received by the editors June 19, 1936.

$\dagger$ Corinna Borden Keen Research Fellow of Brown University.

$\ddagger$ P. Hall, Proceedings of the London Mathematical Society, (2), vol. 36 (1934), p. 90.

$\S$ Defined in $\S 3$. 
THEOREM I. For the ordered set of elements $Q_{1}, Q_{2}, \cdots, Q_{p}$ to constitute a $U$-basis for $G$ it is necessary and sufficient that (a) each element of $G$ be representable in the form $Q_{1}^{x_{1}} Q_{2}{ }^{x_{2}} \cdots Q_{p}^{x_{p}} ;$ (b) the product of the orders of $Q_{1}, Q_{2}, \cdots$, $Q_{\rho}$ equal the order of $G .^{*}$

Let $P_{1}, P_{2}, \cdots, P_{n}$ denote $n$ operations of $G$ whose orders are $g_{1}, g_{2}, \cdots, g_{n}$ respectively. Let $P_{x}$ and $P_{y}$ denote the products $P_{1} x_{1} P_{2} x_{2} \cdots P_{n} x_{n}$ and $P_{1} y_{1} P_{2^{y_{2}}} \ldots P_{n}^{y_{n}}$ respectively, $0 \leqq x_{i}<g_{i}, 0 \leqq y_{i}<g_{i}$. We shall say that $P_{x}$ and $P_{y}$ are formally distinct if at least one $x_{k}$ is not equal to $y_{k}$; we shall call them effectively distinct if they do not represent the same operation of $G$.

Theorem II. For the ordered set of elements $P_{1}, P_{2}, \cdots, P_{n}$ to constitute a $U$-basis for $G$ it is necessary and sufficient that (a) the product $g_{1} g_{2} \cdots g_{n}$ equal the order of $G$; (b) any two formally distinct products $P_{x}$ and $P_{y}$ be effectively distinct.

The following result is often useful:

THEOREM III. If a finite group $G$, of order $g$, contains a set of subgroups $G=G_{1} \supset G_{2} \supset \cdots \supset G_{m}$, each $G_{i+1}$ being a proper subgroup of index $g_{i}$ in $G_{i}$, and if

(a) $G_{m}$ contains a $U$-basis $Q_{1}, Q_{2}, \cdots, Q_{n}$;

(b) $G_{i}-G_{i+1}, i=1,2, \cdots, m-1$, contains an element $P_{i}$ of order $g_{i}$ such that $P_{i}{ }^{{ }^{i}}$ is the lowest power of $P_{i}$ which is in $G_{i+1}$; then the ordered set of elements $P_{1}, P_{2}, \cdots, P_{m-1}, Q_{1}, \cdots, Q_{n}$ (and $Q_{1}, \cdots, Q_{n}, P_{m-1}, \cdots, P_{2}, P_{1}$ as well) constitute a $U$-basis for $G$.

By writing $G_{m-1}$ in cosets with respect to $G_{m}$,

$$
G_{m-1}=G_{m}+P_{m-1} G_{m}+\cdots+P_{m-1}^{o_{m-1}-1} G_{m},
$$

we see from Theorem I that $P_{m-1}, Q_{1}, \cdots, Q_{n}$ form a $U$-basis for $G_{m-1}$. The proof may be completed by induction.

TheOREM IV. If a group $G$ of order $g$ contains two subgroups $H_{1}$ and $H_{2}$, of orders $h_{1}$ and $h_{2}$ respectively; and if

(a) $h_{1} h_{2}=g$

(b) the cross-cut $H_{1} \wedge H_{2}$ is the identity;

(c) $H_{1}$ and $H_{2}$ contain the $U$-bases $P_{1}, \cdots, P_{\rho}$ and $Q_{1}, \cdots, Q_{\sigma}$, respectively; then the ordered set of elements $P_{1}, \cdots, P_{\rho}, Q_{1}, \cdots, Q_{\sigma}$ (or $Q_{1}, \cdots, Q_{\sigma}$, $\left.P_{1}, \cdots, P_{\rho}\right)$ constitute a $U$-basis for $G$.

This theorem is easily proved by writing $G$ in cosets with respect to $H_{1}$ (or $\mathrm{H}_{2}$ ) and applying Theorem I.

* This rather obvious condition is mentioned by Hall for the case of a regular p-group; loc. cit., p. 95 . 
Theorem V. If $G$ is the direct product of the subgroups $G_{1}, G_{2}, \cdots, G_{m}$, and if each subgroup $G_{i}$ has the $U$-basis $Q_{i 1}, \cdots, Q_{i n_{i}}$, then a $U$-basis for $G$ is given by the ordered set $Q_{11}, \cdots, Q_{1_{1}}, Q_{21}, \cdots, Q_{2 n_{2}}, \cdots$, etc.

The wording of the theorem obviously implies that in this ordered arrangement the sets $\left(Q_{i 1}, \cdots, Q_{i n_{i}}\right)$ may be permuted at will, provided that the sequence of the elements within the sets is undisturbed. For $m=2$ this theorem is a corollary of Theorem IV; by induction it can be proved for any $m$.

We conclude this section by giving several examples of groups which have uniqueness-bases.

A. All dihedral groups. For the dihedral group of order $2 m$ (which is generated by two operations $P$ and $Q$ which satisfy the relations $P^{2}=Q^{m}=E$, $\left.Q P=P Q^{-1}\right)$ the ordered set $P, Q$ (and $Q, P$, as well) constitute a $U$-basis.

B. Every symmetric group. By Theorem III we may prove that the ordered set of cycles $a_{1} a_{2}, a_{1} a_{2} a_{3}, \cdots, a_{1} a_{2} \cdots a_{n}$ constitute a $U$-basis for the symmetric group of degree $n$.

C. Every alternating group $A_{n}$. The theorem is obvious for the alternating groups of degrees 2 and 3 . We outline a proof by induction, assuming that the alternating group $A_{n-1}$ of degree $n-1$ has a $U$-basis. There are three cases to consider: (a) when $n$ is odd; (b) when $n$ is divisible by 4 ; (c) when $n$ is divisible by 2 and not by 4 . In case (a) we know that $A_{n}-\mathcal{A}_{n-1}$ contains the cycle $a_{1} a_{2} \cdots a_{n}$. In case (b) it is easy to see that $\mathcal{A}_{n}$ contains the dihedral group of order $n$ as a regular permutation group. In either case (a) or case (b), then, the proof may be completed by using Theorem IV. For case (c) we select from $A_{n}$ the two permutations $s=\left(a_{1} a_{n}\right)\left(a_{2} a_{3}\right)$ and $t=\left(a_{1} a_{2} \cdots a_{n / 2}\right)\left(a_{n / 2+1} \cdots a_{n}\right)$. Now case (c) cannot arise for $n<6$, and it is easy to see that when $n \geqq 6$ all formally distinct products $t^{x} s^{y}$ are effectively distinct, $0 \leqq x<n / 2 ; 0 \leqq y \leqq 1$; except for $x=0$ and $y=0$ the product $t^{x} s^{y}$ will permute the letter $a_{n}$, and hence will not be a permutation in $A_{n-1}$. One may now complete the proof by using Theorem II and the induction-hypothesis.

D. The Sylow p-group $\sum_{p, n}$ of the general $n$-ary linear homogeneous group modulo $p$. It is well known that $\sum_{p, n}$ can be represented by the group of matrices

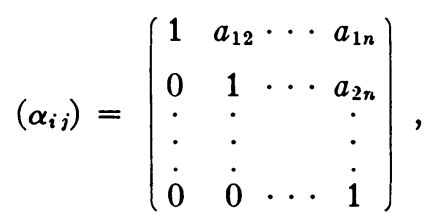

where the $n(n-1) / 2$ coordinates $a_{i j}$ above the main diagonal are arbitrary elements of the Galois field of $p$ elements. If we put $a_{i n}=0, i=1,2, \cdots, n-1$, 
we obtain a representation of $\sum_{p, n-1}$; this group has only the identity in common with the abelian group defined by $a_{i j}=0, j \neq n$. By using Theorem IV and induction over $n$ we may show that a $U$-basis for the group $\left(\alpha_{i j}\right)$ is given by the ordered set

$$
E+e_{12}, E+e_{13}, E+e_{23}, E+e_{14}, \cdots, E+e_{1 n}, E+e_{2 n}, \cdots, E+e_{n-1, n},
$$

where $E$ is the $n$-rowed identity matrix and the $e_{i j}$ are the usual basis-units of the $n$-ary matrix ring.

In conclusion, we offer the quaternion group as the simplest example of a group which has no $U$-basis.

2. In this section we introduce the notion of a normal $U$-basis. A $U$-basis $Q_{1}, Q_{2}, \cdots, Q_{p}$ of a finite group $G$ is said to be normal with respect to $G$ (in short, a normal $U$-basis) if for $i<j ; i=1,2, \cdots, \rho ; j=2,3, \cdots, \rho$, the elements $Q_{a}$ satisfy the $\rho(\rho-1) / 2$ equations

$$
Q_{j} Q_{i}=Q_{i} Q_{j}^{\beta_{j i j}} Q_{j+1}^{\beta_{j i j+1}} \cdots Q_{p}^{\beta_{j i p}} *^{*}
$$

As examples of groups having a normal $U$-basis we mention the dihedral groups and the groups $\sum_{p, n}$ above. Groups which contain a normal $U$ basis evidently constitute an exceptional category, as the restrictions imposed by the definition are relatively strong; for instance, each subgroup $\left\{Q_{j}, Q_{i+1}, \cdots, Q_{p}\right\}$ must be invariant in $G$, and $G$ must be solvable.

An advantageous property of a normal $U$-basis is the following:

THEOREM I. If a finite group $G$ has a normal $U$-basis $Q_{1}, \cdots, Q_{\rho}$, the order of $Q_{i}$ being $g_{i}$, then $G$ is completely defined by the equations

$$
Q_{i}^{o_{i}}=E
$$

and the permutability relations (1) above.

Let $P_{1}, P_{2}, \cdots, P_{\rho}$ be a set of operations; i.e., elements which generate some group, and suppose that these elements are defined by equations (1) and (2) (assuming, of course, that we replace $Q_{i}$ by $P_{i}$ ). Let $\bar{G}$ denote the group generated by $P_{1}, \cdots, P_{\rho}$. Since $\bar{G}$ and $G$ are homomorphic under the correspondence defined by $P_{i} \sim Q_{i}, \dagger$ our theorem will follow if we can show that $G$ and $\bar{G}$ have the same order; that is, if we can show that every product $\Pi=P_{\alpha}{ }^{y_{\alpha}} P_{\beta} y_{\beta} \ldots P_{\alpha}^{z_{\alpha}} \ldots$ of powers of $P_{1}, P_{2}, \ldots, P_{\beta}$ can be brought into the normal form $P_{x}=P_{1}^{x_{1}} P_{2}^{x_{2}} \cdots P_{\rho}^{x_{\rho}}$. by a finite number of reductions, each

* These equations define the commutators $\left(Q_{i}, Q_{i}\right), i<j$. From Theorem I below and the equation $\left(Q_{i}, Q_{i}\right)=\left(Q_{j}, Q_{i}\right)^{-1}$ it follows that $\left(Q_{i}, Q_{j}\right)$ must have the form $Q_{j}{ }^{\xi_{i j}} \cdots Q_{p}{ }^{\xi_{j i \rho}}$.

$\dagger$ Burnside, Theory of Groups of Ginite Order, 2d ed., p. 374. 
reduction consisting of an interchange of two adjacent $P$ 's, using (1), followed by a reduction of exponents by means of (2). This is obviously possible when $\Pi$ contains only $P_{\rho-1}$ and $P_{\rho}$ as factors. The proof may be completed by induction: it is not difficult to show that a product $\Pi$ involving no subscripts less than $k$ can be brought into the normal form by a finite number of reductions, provided that this is true for all products containing subscripts greater than $k$.

Later we shall need the following generalization of the term "normal $U$-basis." Let $G$ be a finite group and let $\Psi$ be a group in which each element is an operator of $G$; suppose, further, that $\Psi$ contains operators* which effect each of the inner isomorphisms of $G$. A $U$-basis $P_{1}, P_{2}, \cdots, P_{\rho}$ of $G$ is said to be normal with respect to $\Psi$ provided that

$$
P_{i} \psi=P_{i}^{\beta_{i} \psi} P_{i+1}^{\beta_{i+1} \psi} \ldots P_{\rho}^{\beta_{\rho} \psi}, \quad i=1,2, \cdots, \rho,
$$

where $\psi$ is a variable operator in $\Psi$.

If for $\Psi$ we take the group $G$ itself, then this definition is equivalent to our earlier definition of a $U$-basis normal with respect to $G$. For in this case equations (3) contain the $\rho(\rho-1) / 2$ equations

$$
P_{i}^{-1} P_{j} P_{i}=P_{i}^{\beta_{j i j}} \ldots P_{\rho}^{\beta_{j i \rho}}, \quad i<j .
$$

THEOREM II. If $\Psi$ is of order $p^{l}$ and if every element of $G$ satisfies the equation $s^{p}=E$, then $G$ contains a $U$-basis normal with respect to $\Psi$.

By taking $\Psi \equiv G$ we have, as a corollary,

THEOREM III. A finite p-group whose elements are of order $p$ (identity excepted) contains a normal $U$-basis.

In connection with Theorem III we observe that each exponent $\beta_{i i j}$ in (4) must be 1 modulo $p$; otherwise, the number of conjugates of $P_{j}$ under $P_{i}$ would contain a factor prime to $p$. Obviously Theorem III is not valid for p-groups in general.

Proof of Theorem II. We write $s^{\prime}$ for $s \psi$, where $s$ and $\psi$ are any elements of $G$ and $\Psi$ respectively. Let $G_{1}$ denote the subgroup of $G$ which is generated by the totality of elements $c_{1}=s^{-1} s^{\prime}$. We define inductively the subgroup $G_{i+1}$. Suppose that $G_{i}$ has already been defined, and suppose that $c_{i}$ represents any element of $G_{i}$. Let $c_{i}^{\prime}$ and $c_{i+1}$ denote $c_{i} \psi$ and $c_{i}^{-1} c_{i}^{\prime}$ respectively, where $\psi$ is any operator of $\Psi$. Then $G_{i+1}$ is defined as the group generated by the totality of elements $c_{i+1}$.

* For a treatment of groups with operators see van der Waerden, Moderne Algebra, vol. I, p. 132.

As concerns its effect on $G$, each operator $\psi$ is equivalent to an operation $T$ in the holomorph of $G$ ( $P_{i} \psi$ and $T^{-1} P_{i} T$ are the same element of $G$ ). It is hardly necessary to point out that $\Psi$ need not be simply isomorphic with a subgroup of the holomorph of $G$. 
Since each $\psi$ effects a $p$-automorphism of $G$, we know that there is associated with a fixed operator $\psi_{\lambda}$ a series of subgroups $G \supset G^{\prime} \supset G^{\prime \prime} \supset \ldots \supset E$, each of index $p$ in the preceding one, such that $s^{(\alpha)} \psi, s^{(\alpha)}$ being any element of $G^{(\alpha)}$, is equal to $s^{(\alpha)}$ multiplied by an element from $G^{(\alpha+1)} .^{*}$ From this we see at once that $G_{i+1}$ is a proper subgroup of $G_{i}, i=1,2, \cdots$; consequently, the series $G=G_{0} \supset G_{1} \supset G_{2} \supset \cdots$ must terminate in the identity $E$. Suppose that $G_{f} \equiv E$, but $G_{f-1} \not \equiv E$. We shall say that $G$ is of class $f$ with respect to $\Psi$. (By hypothesis, $\Psi$ contains operators which bring about each of the inner isomorphisms of $G$. Hence $G_{1}$ contains the commutator subgroup of $G$. And if $\Psi$ is $G$ itself, then our definition of class coincides with the usual one.)

Now the group $G_{f-1}$ of order $p^{n_{f-1}}$, say, is abelian and of type $1,1, \cdots, 1$. For $G_{f-1}$ we can construct a $U$-basis $P_{f-1,1}, P_{f-1,2}, \cdots$, and this $U$-basis will be normal with respect to $\Psi$, since every element of $G_{f-1}$ satisfies the equation $c \psi=c . \dagger$

If $G$ is of class 1 with respect to $\Psi$, then our construction is at an end. Otherwise, we proceed by induction over $G_{i}$. Suppose that for $G_{k+1}$ we have already constructed a $U$-basis normal with respect to $\Psi$. Now each quotientgroup $G_{i} / G_{i+1}$, of order $p^{n_{i}}$, is abelian and of type $1,1, \cdots, 1$. Hence we may construct for $G_{k} / G_{k+1}$ a $U$-basis $u_{1}, u_{2}, \cdots, u_{n_{k}}$. From each of those cosets of $G_{k}$ (with respect to $G_{k+1}$ ) which correspond to $u_{1}, u_{2}, \ldots$ we select an element as a representative, obtaining thereby $n_{k}$ elements $P_{k \lambda}, \lambda=1,2, \cdots, n_{k}$. The ordered set $P_{k 1}, \cdots, P_{k n_{k}}$ followed by the elements of the $U$-basis for $G_{k+1}$ (in the proper sequence) will constitute a $U$-basis for $G_{k}$ which is normal with respect to $\Psi$. This assertion can readily be proved by using Theorem I of $\S 1$, together with the fact that the order of $G_{k}$ is $P^{r_{k}}$, where $r_{k}$ equals $\sum_{\alpha-k}^{f-1} n_{\alpha}$.

The construction which we have just given leads to a $U$-basis containing exactly $m$ elements, where $p^{m}$ is the order of $G$. It may be pointed out that every $U$-basis of $G$, normal or not, must contain exactly $m$ elements. This follows from two considerations: every finite group whose elements are of order $p$, identity excepted, is a regular $p$-group; $\ddagger$ the number of elements in a $U$-basis for a regular $p$-group is an invariant of the group.

3 . In the introduction we mentioned an important category of $p$-groups, which resemble the abelian $p$-groups in that any two $U$-bases have the same number of elements of a given order. These are the regular $p$-groups, which have been defined in the following way:§ the $p$-group $G$ will be called regular

* Miller, Blichfeldt, and Dickson, Finite Groups, p. 136.

$\dagger$ The proof of Theorem II depends mainly upon familiar properties of abelian groups $A$ of order $p^{m}$ and type $1,1, \cdots, 1: A$ has a $U$-basis, and the number of elements in every $U$-basis is exactly $m$.

$\ddagger$ Hall, loc. cit., p. 74 .

§ Hall, loc: cit., p. 73. 
if, given any positive integer $\alpha$ and a pair of elements $P$ and $Q$ of $G$, it is always possible to find elements $S_{3}, S_{4}, \cdots, S_{\rho}$ all belonging to the commutator subgroup of $\{P, Q\}$ and satisfying the equation $(P Q)^{p^{\alpha}}=P^{p^{\alpha}} Q^{p^{\alpha}} S_{3}^{p^{\alpha}} S_{4}^{p^{\alpha}} \ldots$ $S_{p}^{p^{\alpha}}$.

For an understanding of what follows, one must keep well in mind certain definitive properties of a regular $p$-group:

(a) The $p^{\alpha}$ th powers of the elements of $G$ constitute a characteristic subgroup $\mho_{\alpha}(G)$.

(b) Those elements in $G$ whose orders divide $p^{\beta}$ constitute a characteristic subgroup $\Omega_{\beta}(G)$.

(c) The group $G$ is conformal with an abelian group $A$.

(d) The orders of the elements in any $U$-basis of $G$ are the same as the invariants $p^{\epsilon_{1}}, p^{\epsilon_{2}}, \cdots, p^{e_{r}}$ of $A$. (These orders have been called the typeinvariants of $G$.)

(e) If $Q_{x}$ is an element $Q_{1}{ }^{x_{1}} Q_{2}{ }^{x_{2}} \ldots Q_{r}^{x_{r}}$, written in the normal form with respect to the $U$-basis $Q_{1}, Q_{2}, \cdots, Q_{r}$, then the order of $Q_{x}$ is equal to the order of its constituent $Q_{\lambda}{ }_{\lambda}$, of highest order.

(f) If $P$ and $Q$ are any two elements of $G$, then the order of every element in the commutator subgroup of $\{P, Q\}$ divides the order of $P$ (and of $Q$ ) relative to the central of $G$.

From (a) and (b) it is clear that $G$ contains two series of characteristic subgroups: $G=\mho_{0} \supset \mho_{1} \supset \cdots \supset \mho_{\delta}=E ; E=\Omega_{0} \subset \Omega_{1} \subset \cdots \subset \Omega_{\delta}=E$, where $p^{\delta}$ is the order of an element of highest order in $G$. From (c) it follows that $\delta$ is equal to the largest one of the $\epsilon$ 's in (d).

(g) The order $p^{\omega_{\alpha}}$ of $\Omega_{\alpha} / \Omega_{\alpha-1}$ equals the order of $\mho_{\alpha-1} / \mho_{\alpha}, \alpha=1,2, \cdots, \delta$. In particular, $G / \mho_{1}$ is of order $\omega_{1}$. The $\omega$ 's satisfy the inequalities $\omega_{1} \geqq \omega_{2} \geqq \cdots \geqq \omega_{\delta}$. Furthermore, $\omega_{1}$ equals $r$, the number of type-invariants of $G$.

(h) For the $\epsilon$ 's and $\omega$ 's we have the relation $\sum_{i=1}^{r} \epsilon_{i}=\sum_{j=1}^{r} \omega_{j}=m$, where $p^{m}$ is the order of $G$ (and of $A$ ).

(i) $\mho_{\alpha}(G)$ and $\Omega_{\beta}(G)$ are conformal with $\mho_{\alpha}(A)$ and $\Omega_{\beta}(A)$ respectively, $\alpha, \beta=0,1, \cdots, \delta$.

Let $V_{i}(G)$ denote the cross-cut $\mho_{1}(G) \wedge \Omega_{i}(G), i=0,1, \cdots, \delta$, and let $W_{i}(G)$ denote the group $\left\{V_{i}(G), \Omega_{i-1}(G)\right\}$.

(j) The groups $V_{i}(G), W_{i}(G), \Omega_{i}(G) / V_{i}(G)$, and $\Omega_{i}(G) / W_{i}(G)$ are conformal respectively with the groups which are obtained by replacing $G$ with $A$.

(k) If the exponents of the invariants of $A$, arranged in descending order of magnitude, are given by $\delta_{1} \geqq \delta_{2} \geqq \cdots \geqq \delta_{r}$, and if in this arrangement the $\delta$ 's constitute $s$ sets, the $j$ th set consisting of $h_{j}$ equal $\delta$ 's having the common 
value $e_{i}$, then the order of $\Omega e_{j}(A) / W e_{j}(A)$ is $p^{h_{j}}$. Obviously $m=\sum_{i=1}^{r} \delta_{i}$ $=\sum_{j=1}^{s} h_{j} e_{j}$.

Items (a) through (i) are given explicitly in the paper of Hall to which reference has already been made (see pp. 73-81); item ( $j$ ) is contained implicitly in Hall's results; item (k) is a familiar result from the theory of abelian p-groups.

Definition: $A U$-basis $P_{1}, P_{2}, \cdots, P_{r}$ for a regular $p$-group $G$, the order of $P_{i}$ being $p^{\delta_{i}}$, is said to be $\omega$-normal provided that

(1) $\delta_{1} \geqq \delta_{2} \geqq \cdots \geqq \delta_{r}$

(2) when $\left(P_{j}, P_{i}\right),{ }^{*} i<j$, is expressed in the normal form

$$
\left(P_{j}, P_{i}\right)=P_{1}^{\alpha_{j i 1}} P_{2}^{\alpha_{j i 2}} \cdots P_{r}^{\alpha_{j i r}}, \quad i=1,2, \cdots, r ; j=2,3, \cdots, r,
$$

each $\alpha_{j i k}$ is divisible by $p$ for $k \leqq j$.

We state without proof two implications of this definition:

(3) for $k<j, \alpha_{j i k} \equiv 0 \bmod p^{\delta_{k}-\delta_{j}}($ see (e) and (f));

(4) if the normal form of $\left(P_{i}, P_{j}\right), i<j$, is given by $P_{1}{ }^{\alpha_{i j 1}} \cdots P_{r}{ }^{\alpha_{i j r}}$, then the highest power of $p$ that divides $\alpha_{i j k}$ also divides $\alpha_{j i k}$, and conversely. Moreover, (2) is clearly a consequence of (1) when no two of the $\delta$ 's a are equal.

For the abelian group $A$ conformal with $G$ any $U$-basis for which (1) is satisfied may be regarded as a "normal form," since all such $U$-bases are equivalent under the holomorph of $A$. In defining a normal form for a $U$-basis of $G$ we must obviously demand more, and (2) seems to be the most natural additional requirement which can be satisfied in the case of exery regular $p$-group. The qualifying phrase " $\omega$-normal" is suggested by the fact that $P_{1}, P_{2}, \cdots, P_{r}$, regarded as representatives of a $U$-basis for $G / \mho_{1}$, constitute a normal $U$-basis for this quotient group; i.e.,

$$
P_{j} P_{i} \equiv P_{i} P_{j} P_{j+1}^{\alpha_{j i j+1}} \cdots P_{r}^{\alpha_{j i r}} \bmod \mho_{1} .
$$

As an attempt at defining a normal form for a $U$-basis, our definition above is obviously of no value unless we can prove the following theorem:

Every regular p-group $G$ contains an w-normal $U$-basis.

First, we explain a method for constructing a set of elements which satisfy requirements (1) and (2) above. To avoid repeated explanations, the symbols $G, \Omega(), m, h_{j}, e_{j}$, etc., will have the same significance as in (a) through (k) above.

* This is the familiar notation for the commutator $P_{j}^{-1} P_{i}^{-1} P_{j} P_{i}$. 
Let $\Omega_{a}(G)$ be the first term in the series $E=\Omega_{0} \subset \Omega_{1} \subset \cdots \subset \Omega_{\delta}=G$ for which $W_{a}$ is a proper subgroup of $\Omega_{a}$; i.e.,

$$
W_{j} \equiv \Omega_{i}, \quad j=0,1, \cdots, a-1 ; \quad W_{a} \subset \Omega_{a} .
$$

From (j) and (k) we see that the order of $\Omega_{a} / W_{a}$ is exactly $p^{h_{a}}$. Furthermore, every element of this quotient-group is of order $p$, except for the identity. Since $\Omega_{a}$ and $W_{a}$ are characteristic subgroups of $G$, and since each element of $G$ effects an automorphism of $\Omega_{a} / W_{a}$, we can construct for this quotient-group a $U$-basis, $u_{1}, u_{2}, \cdots, u_{h_{s}}$, say, which is normal with respect to $G$ (see Theorem II of $\$ 2$ ). From the coset of $\Omega_{a}$ which corresponds to $u_{j}$ we select any element $Q_{i}$ as a representative, obtaining thereby the $h_{s}$ elements $Q_{1}, Q_{2}, \cdots, Q_{h_{i}}$.

(6) Each $Q_{j}, j=1,2, \cdots, h_{s}$, is of order $p^{a}$.

Otherwise, contrary to (5), we could find a $k<a$ for which $W_{k} \not \equiv \Omega_{k}$.

It is easy to see that every element of $\Omega_{a}$ can be expressed in the form $Q_{1} x_{1} Q_{2}{ }^{x_{3}} \cdots Q_{h_{a}}^{x_{h_{2}} w_{a, x}}$, where $w_{a, x}$ is an element in $W_{a}$. And since the $Q_{j}$ 's are representatives of a $U$-basis for $\Omega_{a} / W_{a}$ which is normal with respect to $G$, it is clear that for a variable element $X$ in $G$ we have the congruences

$$
X^{-1} Q_{j} X \equiv Q_{j} Q_{j+1}^{\xi_{j+1}} \cdots Q_{h_{a}}^{\xi_{h}} \bmod W_{a}, \quad j=1,2, \cdots, h_{s} .
$$

From (i), (j), and (k) we also have the equality

$$
a=e_{s} .
$$

If $\Omega_{a}$ is $G$ itself, then our construction is at an end. If not, let $\Omega_{b}$ be the first term in the series $\Omega_{a+1}, \Omega_{a+2}, \cdots$ for which

$$
W_{b} \subset \Omega_{b} ; W_{a+l} \equiv \Omega_{a+l}, \quad l=0,1, \cdots, b-a-1 .
$$

As above, we construct for $\Omega_{b} / W_{b}$, which is necessarily of order $p^{h_{s-1}}$, a $U$-basis normal with respect to $G$, and from each coset of $\Omega_{b}$ which corresponds to one of these basis-elements we choose an element, obtaining the $h_{s-1}$ elements $R_{1}, R_{2}, \cdots, R_{h_{t-1}}$.

(10) Each of the $R$ 's is of order $p^{b}$.

Suppose that one of them, $R_{\lambda}$, say, were of order less than $p^{b}$. Then $R_{\lambda}$ would necessarily occur among the elements of a certain set $\Omega_{k}-W_{k}$ where $k<b$. From (5) and (9) we know that $a$ is the only value of $k<b$ for which $W_{k} \subset \Omega_{k}$. And certainly $R_{\lambda}$ cannot be an element in $\left\{\mho_{1}, \Omega_{a}\right\}$, since $\Omega_{a}$ is contained in $W_{b}$. As in (8) above, we have the equality $b=e_{s-1}$.

From the manner of their construction it follows that the $R$ 's satisfy congruences of the type 


$$
X^{-1} R_{i} X=R_{i} R_{i+1}^{\xi_{i+1}} \cdots R_{h_{s-1}}^{\xi_{h_{s}-1}} \bmod W_{b}, \quad i=1,2, \cdots, h_{s-1},
$$

when $X$ is any element of $G$.

Since $W_{b}$ is a subgroup of $\left\{\mho_{1}, \Omega_{a}\right\}$, we may replace (11) by

$$
X^{-1} R_{i} X \equiv R_{i} R_{i+1}^{\xi_{i+1}} \cdots R_{h_{s-1}}^{\xi_{h_{-1}-1}} Q_{1}^{\eta_{1}} \cdots Q_{h_{s}}^{\eta_{i}} \bmod \mho_{1} .
$$

If $\Omega_{b}$ is not equal to $G$, then we continue the construction; and at this point it is reasonably clear how the construction advances. The final (the $s$ th) stage will consist of selecting $h_{1}$ elements $P_{1}, P_{2}, \cdots, P_{h}$ of $\Omega_{\delta} \geqq G$ which correspond to a $U$-basis of $G / W_{\delta}$, this $U$-basis being, of course, normal with respect to $G$. Thus we obtain an ordered set of $r=\sum_{i=1}^{s} h_{i}$ elements

$$
P_{11}, P_{12}, \cdots, P_{1 h_{1}}, P_{21}, \cdots, P_{s-11}, \cdots, P_{s 1}, \cdots, P_{s h_{s}},
$$

where $P_{s-1 i}$ and $P_{s j}$ denote the elements $R_{i}$ and $Q_{j}$ above.

For our purpose the significant properties of these elements are the two following: $\delta_{1} \geqq \delta_{2} \geqq \cdots \geqq \delta_{r}$, and

$$
X^{-1} P_{j} X \equiv P_{j} P_{j+1}^{\xi_{j+1}} \cdots P_{r}^{\xi_{r}} \bmod \mho_{1}(G), \quad j=1,2, \cdots, r .
$$

(For the sake of a simpler notation we have replaced $P_{11}$ by $P_{1}, P_{12}$ by $P_{2}, \cdots$, $P_{s h}$ by $P_{r}$.) It is clear, therefore, that if the elements $P_{1}, P_{2}, \cdots, P_{r}$ form a $U$-basis for $G$, then this $U$-basis will be $\omega$-normal.

To prove that $P_{1}, P_{2}, \cdots, P_{r}$ constitute a $U$-basis, it is sufficient to show that they form a canonical basis for $G$, since it is known that every canonical basis of a regular $p$-group is necessarily a $U$-basis. ${ }^{*}$

A canonical-basis of a regular $p$-group has been defined* as a set of $\omega\left(=\omega_{1}\right)$ elements $Q_{1}, Q_{2}, \cdots, Q_{\omega}\left(\omega\right.$ being the order of $\left.G / \mho_{1}(G)\right)$ which satisfy the following conditions:

$(\alpha)$ there exists a set of $\omega$ subgroups $G=K_{1} \supset K_{2} \supset \cdots \supset K_{\omega} \supset K_{\omega+1}=\mho_{1}$, each being invariant in $G$ and a proper subgroup of the preceding, such that each of the $\omega$ sets $K_{i}-K_{i+1}$ contains exactly one of the $Q_{i}$ 's;

$(\beta)$ the product of the orders of the $Q_{i}$ 's is as small as possible, consistent with $(\alpha)$.

It is known that

$(\gamma)$ the product of the orders of the elements in and canonical basis must equal the order of $G . \dagger$

To show that the elements $P_{1}, \cdots, P_{r}$ above form a canonical-basis, it is therefore sufficient to show that they satisfy requirements $(\alpha)$ and $(\gamma)$.

* Hall, loc. cit., p. 91.

$\dagger$ Hall, loc: cit., p. 92. 
Now $(\gamma)$ is satisfied, since the elements (13) constitute $s$ sets, the $j$ th set containing $h_{j}$ elements each of order $p^{e_{j}}$ [see (k) and (8) above].

To prove that $(\alpha)$ is also satisfied, we observe from (14) that the group $F_{i}=\left\{P_{i}, P_{i+1}, \cdots, P_{r}, \mho_{1}\right\}$ is an invariant subgroup of $G$; moreover $F_{i}$ is a proper subgroup of index $p$ in $F_{i-1}$, and $r$ is equal to $\omega$. Hence the series $G=F_{1} \supset F_{2} \supset \ldots \supset F_{r} \supset \mho_{1}$ has the properties of the $K$-series in $(\alpha)$; and since $F_{i}-F_{i+1}$ contains $P_{i}$ and no other one of the $P$ 's, it follows that requirement $(\alpha)$ is satisfied by the $r$ elements $P_{1}, P_{2}, \cdots, P_{r}$.

Whenever an $\omega$-normal $U$-basis is normal with respect to $G$, that is, whenever the congruences $\left(P_{j}, P_{i}\right) \equiv P_{j+1}^{\theta_{j i j+1}} \ldots P_{r}^{\theta_{j i r}} \bmod \mho_{1}, i<j$, can be replaced by equalities

$$
\left(P_{j i} P_{i}\right)=P_{j+1}^{\lambda_{i j i+1}} \cdots P_{r}^{\lambda_{j i r}},
$$

then, from Theorem I of $\S 2$, we know that $G$ is completely defined by the orders $p^{\delta_{1}}, \cdots, p^{\delta_{r}}$ and the exponents in (15). The existence of a $G$-normal $U$-basis is clearly exceptional and it is an open question whether the data provided by an $\omega$-normal $U$-basis, namely, the orders of the elements and the $r(r-1) / 2$ equations (2), are always sufficient to define the regular $p$-group from which they are derived.

A Note on $l$-Matrices. ${ }^{*}$ Let $\left(\xi_{i j}\right)$ be an $r$-rowed square matrix whose coordinates are arbitrary rational integers, and let $\delta_{1}, \delta_{2}, \cdots, \delta_{r}$ be a sequence of fixed positive integers satisfying the inequalities $\delta_{1} \geqq \delta_{2} \geqq \cdots \geqq \delta_{r}$. The $l$ matrix $\left(x_{i j}\right)$ we shall define as the matrix formed by replacing each $\xi_{i j}$ by the set of integers having the form $\xi_{i j} \pm \lambda_{i j} p^{\delta_{i}}$; that is, $\left(x_{i j}\right)$ is the matrix whose $j$ th column is composed of residue classes $\left[\xi_{i j}\right]$ modulo $p^{\delta_{i}}$. The class $\left[\xi_{i j}\right]$ is characterized by the least positive residue of $\xi_{i j}$ modulo $p^{\delta_{j}}$; accordingly, we shall usually assume that the coordinate $x_{i j}$ in the $l$-matrix $\left(x_{i j}\right)$ is a least positive residue rather than a class $\left[\xi_{i j}\right]$. Two $l$-matrices are naturally to be regarded as distinct unless their corresponding coordinates are identical. The totality of distinct $l$-matrices constitute a set, which we shall designate by the expression $L_{p}\left(\delta_{1}, \delta_{2}, \cdots, \delta_{r}\right)$. The sum of two $l$-matrices $\left(x_{i j}\right)$ and $\left(y_{i j}\right)$ we shall define as the $l$-matrix $\left(z_{i j}\right)$ for which $z_{i j}$ is the least positive residue of $x_{i j}+y_{i j}$ modulo $p^{\delta_{i}}, j=1,2, \cdots, r$; the product $\left(x_{i j}\right)\left(y_{i j}\right)$ is the $l$-matrix $\left(w_{i j}\right)$ in which $w_{i j}$ is the least positive residue of $\sum_{\alpha=1}^{r} x_{i \alpha} y_{\alpha j}$ modulo $p^{\delta_{j}}$. Those $l$-matrices for which the conditions

and

( $\alpha) x_{i j} \equiv 0 \bmod p^{\delta_{j}-\delta_{i}}, i>j$

( $\beta)\left|x_{i j}\right| \not \equiv 0 \bmod p$

* These $l$-matrices were first defined, in a slightly different form, by A. Ranum, these Transactions, vol. 8 (1907), pp. 71-91. 
hold constitute under multiplication a group, which we shall refer to as "the group of $l$-matrices."* This group, which we shall denote by the expression $G L_{p}\left(\delta_{1}, \delta_{2}, \cdots, \delta_{r}\right)$, is simply isomorphic with the group of isomorphisms of the abelian $p$-group of type $\delta_{1}, \delta_{2}, \cdots, \delta_{r}{ }^{*}$

Multiplication is not, in general, assoc ative for any three matrices of the set $L_{p}\left(\delta_{1}, \cdots, \delta_{r}\right)$. For $l$-matrices $\left(x_{i j}\right)$ which satisfy $(\alpha)$, however, multiplication is associative and distributive, and these $l$-matrices constitute a ring. Thus any expression $\left(a\left(x_{i j}\right)+b\left(y_{i j}\right)+\cdots\right)^{n}\left(c\left(u_{i j}\right)+d\left(v_{i j}\right)+\cdots\right)^{m} \cdots$, where $a, b, m, n$ are positive integers, defines a unique $l$-matrix, and this consideration is the justification for our later notation. In particular, the $l$-matrix defined by the expression $a\left(x_{i j}\right)$, where $\left(x_{i j}\right)$ satisfies condition $(\alpha)$, may be regardod as $\left(x_{i j}\right)+\left(x_{i j}\right)+\cdots+\left(x_{i j}\right)$, where there are $a$ terms, as $\left(\alpha_{i j}\right)\left(x_{i j}\right)$, or as $\left(x_{i j}\right)\left(\alpha_{i j}\right)$, where $\left(\alpha_{i j}\right)$ is the $l$-matrix whose diagonal elements are the least positive residues of $a$ modulis $p^{\delta_{1}}, p^{\delta_{2}}, \cdots$, etc., the remaining elements being zeros.

4. This section is concerned with applying the data furnished by an $\omega-$ normal $U$-basis to the problem of constructing a one-to-one representation by $l$-matrices $\dagger$ for a special category of regular $p$-groups; that is, the regular $p$-groups of class $2 . \ddagger$ The theory of representations of a group of order $g$ by means of matrices with coefficients in a field of characteristic prime to $g$ has been rather thoroughly exploited. Little is known, however, about representations of $p$-groups by matrices with coefficients in a field of characteristic $p$; and it is fair to say that the problem of representing a given $p$-group by $l$-matrices has received almost no attention. $\S$

Since the group $G L_{p}\left(\delta_{1}, \cdots, \delta_{r}\right)$-the group of $l$-matrices-is simply isomorphic with the group of automorphisms of the abelian p-group of type $\delta_{1}, \delta_{2}, \cdots, \delta_{r}$, we can easily construct a representation of a $p$-group $G_{p}$ if we can find an abelian p-group $A_{p}$ which is transformed into itself by $G_{p}$. Thus we can always construct a multiply-isomorphic representation by $l$-matrices for any $p$-group $G_{p}$, whether regular or not, since $G_{p}$ always contains invariant abelian subgroups. The real difficulty arises when we demand a 1-1 representation of $G_{p}$ (that is, a representation which is simply isomorphic with $G_{p}$ ).

* Ranum, pp. 84-85.

$\dagger$ These $l$-matrices are defined in $\$ 3$.

$\ddagger$ Groups of class 2 (metabelian groups, in the terminology of American mathematicians) were originally defined as groups having abelian central quotient-groups (W. B. Fite, Proceedings of the American Association for the Advancement of Science, vol. 49 (1901), p. 41). They have also been defined as groups having abelian commutator subgroups. The two definitions are obviously equivalent.

$\S$ The reciprocal problem, namely, the investigation of the subgroups of the group $G L_{p}\left(\delta_{1}, \delta_{2}, \cdots, \delta_{r}\right)$ has been widely discussed. 
It is precisely these 1-1 representations which are of most interest, and in the case of regular $p$-groups of class 2 a method for constructing them may be developed from the theory of regular permutation groups.

Let $G$ be a regular $p$-group of class 2 and of order $p^{m}, p>2,{ }^{*}$ which is represented as a regular $\dagger$ permutation group on its $p^{m}$ elements. Let $K(G)$ denote the holomorph of $G$, and let $H$ denote that representation in $K(G)$ of the group of inner isomorphisms of $G$ whose permutations omit the symbol for the identity of $G$. Let $s_{1}, s_{2}, \cdots, s_{p^{m}}$ denote the permutations of $G$, and let $S_{i}$ denote that permutation of $H$ which transforms $G$ according to $s_{i}$. Since $G$ is of class 2, its commutator subgroup $C(G)$ is contained in its central $\Gamma(G)$; furthermore, $H$ is abelian, since it is simply isomorphic with $G / \Gamma$. From this we see that $G$ is multiply isomorphic with $H$ under the correspondence defined by $s_{i} \sim S_{i}{ }^{\lambda}, i=1,2, \cdots, p^{m}$, where $\lambda$ is any fixed integer.

At this point we introduce several useful formulas, which one may readily verify:

(1) $\left(s_{i}, s_{j}\right)=\left(s_{i}^{-1}, s_{j}^{-1}\right)=\left(s_{i}^{-1}, s_{i}\right)=\left(s_{j}, s_{i}^{-1}\right)=\left(s_{j}, s_{i}\right)^{-1}$,

(2) $\left(s_{i}^{x}, s_{j}^{y}\right)=\left(s_{i}, s_{j}\right)^{x y}$,

(3) $\left(s_{i}, s_{j}\right)=\left(S_{i}, s_{j}\right)=\left(s_{i}, S_{j}\right) ;\left(S_{i}, S_{j}\right)=E$.

Let $p^{\mu}$ be the order of the element of highest order in $H$. Since $p$ is an odd prime $\left(p^{\mu}-1\right) / 2$ is a positive integer; and we denote this integer by the letter $a$.

We shall need the following results: $\ddagger$

(4) The $p^{m}$ products $S_{i}{ }^{a} S_{i}$ constitute a regular permutation group $G_{a}$ which is abelian and conformal with $G$.

(5) The cross-cut $G \wedge G_{a}$ is the permutation group $\Gamma$.

(6) The group $G_{a}$ is transformed into itself by $G$, and conversely.

Let $K\left(G_{a}\right)$ denote the holomorph of $G_{a}$, written as a permutation group on the letters of $G$, and let $I\left(G_{a}\right)$ be that representation in $K\left(G_{a}\right)$ of the group of isomorphisms of $G_{a}$ which omits the symbol for the identity of $G$. Correspondingly, we define $I(G)$ as that representation in $K(G)$ of the group of isomorphisms of $G$ which omits the symbol for the identity of $G$.

(7) The permutation group $I(G)$ is a subgroup of $I\left(G_{a}\right)$.

(8) Between the permutations $t_{1}, t_{2}, \ldots$ of $G_{a}$ and those of $G$ there is a

* The assumption $p>2$ is pertinent, since a group of order $2^{m}$ is regular only when it is abelian.

$\dagger$ The simultaneous occurrence of "regular" in two distinct and unrelated meanings is unfortunate; both usages, however, are already established in the literature. To avoid confusing repetitions of the adjective "regular", we agree that throughout the remainder of this section the symbol $G$ shall be used precisely in the sense above.

$\ddagger$ These Transactions, vol. 37 (1935), pp. 163-171. This paper will be referred to as $H$. 
1-1 correspondence, which is defined by the equations $t_{i}=S_{i}{ }^{a} s_{i}$ and $s_{i}=S_{i}^{-a} t_{i}$.

When, in the future, we speak of an element of $G$ (or of $G_{a}$ ) as the "corresponding element" of an element in $G_{a}$ (or in $G$ ), we shall have in mind the correspondence defined by the equations in (8).

Let $P_{1}, P_{2}, \cdots, P_{r}$, of orders $p^{\delta_{1}}, p^{\delta_{2}}, \cdots, p^{\delta_{r}}$, be the permutations of an $\omega$-normal $U$-basis for $G$; i.e., a set of permutations satisfying the conditions

$$
\begin{aligned}
P_{i}^{p \delta_{i}} & =E, \quad \delta_{1} \geqq \delta_{2} \geqq \cdots \geqq \delta_{r} ; \\
\left(P_{j}, P_{i}\right) & =P_{1}^{\alpha_{j i 1}} P_{2}^{\alpha_{j i 2}} \cdots P_{r}^{\alpha_{j i r}}, \quad i<j ; i=1,2, \cdots, r ; j=2,3, \cdots, r ; \\
& \alpha_{j i k} \equiv 0 \bmod p, \text { for } k \leqq j .
\end{aligned}
$$

Let $\bar{S}_{i}, i=1,2, \cdots, r$ denote the permutation of $H$ which transforms $G$ according to $P_{i}$.

Of fundamental importance for our method of attack is the following result:*

(11) The permutations $A_{1}={\overline{S_{1}}}_{1}{ } P_{1}, A_{2}=\bar{S}_{2}{ }^{a} P_{2}, \cdots, A_{r}=\bar{S}_{r}{ }^{a} P_{r}$, which are necessarily of orders $p^{\delta_{1}}, p^{\delta_{2}}, \cdots, p^{\delta_{r}}$ respectively, constitute a $U$-basis for $G_{a}$.

In what follows we shall usually write $T_{i}$ for $\bar{S}^{-a}$. From the equation

$$
P_{i}=T_{i} A_{i}
$$

it follows that each basis-element $P_{i}$ may be regarded as a product of a basiselement of $G_{a}$ by an automorphism of $G_{a}$, and this is a point of view which we shall constantly emphasize.

From (1), (2), and (3) it is easy to establish the following formulas:

$$
\begin{aligned}
& \left(A_{j}, T_{i}\right)=\left(A_{i}, P_{i}\right)=\left(\bar{S}_{j}^{a} P_{i}, P_{i}\right)=\left(P_{i}, P_{i}\right)^{a+1} ; \\
& \left(A_{i}, T_{j}\right)=\left(A_{j}, T_{i}\right)^{-1} ;\left(A_{i}, T_{i}\right)=E .
\end{aligned}
$$

Now each $T_{i}, i=1,2, \cdots, r$, is a permutation in the group of isomorphisms of $G_{a}$ (see (7) above). Regarded as an automorphism of $G_{a}$, each $T_{i}$ is equivalent to a change of basis for $G_{a}$, defined by $r$ equations of the form

$$
\left\{\begin{array}{c}
A_{1} \rightarrow A_{1}^{\prime}=A_{1}^{1+\gamma_{1 i 1}} A_{2}^{\gamma_{1 i 2}} \cdots A_{r}^{\gamma_{1 i r}} \\
\cdot \cdot \cdot \cdot \cdot \cdot \cdot \cdot \cdot \cdot \cdot \cdot \cdot \cdot \cdot \cdot \cdot \cdot \cdot \cdot \cdot A_{r}^{\gamma_{r i 1}} A_{2}^{\gamma_{r i 2}} \cdots A_{r}^{1+\gamma_{r i r}} \\
A_{r} \rightarrow A_{r}^{\prime}=A_{1}^{2}
\end{array}\right.
$$

where $\gamma_{j i k}$ is a least positive residue modulo $p^{\delta_{k}}$.

If we denote the matrix of the exponents in (15) by $E_{r}+M_{i}, E_{r}$ being the identity matrix of $G L_{p}\left(\delta_{1}, \delta_{2}, \cdots, \delta_{r}\right)$, then the $r$ matrices $E_{r}+M_{i}$ generate

* H, p. 177. 
a 1-1 representation of $H$ (and consequently a $p^{\gamma-1}$ representation of $G$, where $p^{\gamma}$ is the order of the central $\Gamma(G)$ ). We know that each $\gamma_{j i k}$ in (15) is uniquely determined by the permutations $T_{i}$ and $A_{1}, A_{2}, \cdots, A_{r}$. What is of equal interest, perhaps, is the fact that the $\gamma_{j i k}$ are uniquely determined by equations (9) and (10) together with the equations

$$
\left(P_{j}, P_{i}\right) P_{k}=P_{k}\left(P_{j}, P_{i}\right), \quad i, j, k=1,2, \cdots, r,
$$

for the reason that any $r$ operations which satisfy (9), (10), and (16) generate a group which is simply isomorphic with $G{ }^{*}$

We indicate a method for computing the $\gamma_{j i k}$ from the data in (9), (10), and (16). If $T_{k}^{x}=E, k=1,2, \cdots, r$, where $x$ ranges over all the exponents $\alpha_{j i k}$ in (10), that is, if each constituent $P_{k}^{\alpha_{i j k}}$ of $\left(P_{j}, P_{i}\right)$ is in $\Gamma$, then it follows from (12) and (13) that $\gamma_{j i k}$ is equal to the least positive residue of $(a+1) \alpha_{j i k}$ modulo $p^{\delta_{k}}$. In general, however, it is impossible to find for $G$ an $\omega$-normal $U$-basis for which this favorable situation arises. The following procedure is always valid. In (13) we replace each $P_{k}$ by $T_{k} A_{k}$, obtaining thereby a first approximation for $\left(A_{i}, T_{i}\right)$ in terms of the basis elements of $G_{a}$ :

$$
\begin{aligned}
\left(A_{i}, T_{i}\right) & =\left(P_{1}^{\alpha_{j i 1}} \cdots P_{r}^{\alpha_{j i r}}\right)^{a+1} \\
& =\left(T_{1}^{\alpha_{j i 1}} A_{1}^{\alpha_{j i 1}} T_{2}^{\alpha_{j i 2}} A_{2}^{\alpha_{j i 2}} \cdots T_{r}^{\alpha_{j i r}} A_{r}^{\alpha_{j i r}}\right)^{a+1}=T_{\alpha}^{a+1} A_{\alpha}^{a+1} c_{\alpha}^{a+1},
\end{aligned}
$$

where

$$
\begin{aligned}
T_{\alpha}=T_{1}^{\alpha_{j i 1}} \cdots T_{r}^{\alpha_{j i r}} ; A_{\alpha}=A_{1}^{\alpha_{j i 1}} \cdots A_{r}^{\alpha_{j i r}} ; c_{\alpha}=\prod_{k, l}\left(A_{k}, T_{l}\right)^{\alpha_{j i k} \alpha_{j i l}}, \\
k<l ; k=1,2, \cdots, r ; l=2,3, \cdots, r .
\end{aligned}
$$

Since $\left(P_{j}, P_{i}\right)$ is in the central of $G$, we know that $T_{\alpha}$ must be the identity of $H$. We observe, in addition, that for $k \leqq j$ the order of $\left(A_{k}, T_{l}\right)^{\alpha_{j i k} \alpha_{j i l}}$ is less than the order of $\left(A_{k}, T_{l}\right)$, since $\alpha_{j i k}$ is divisible by $p$ for $k \leqq j$; and for $k>j$, the first constituent of $\left(A_{k}, T_{l}\right)=P_{1} \alpha_{k l 1} \ldots P_{r} \alpha_{k l r}$ whose exponent is prime to $p$ must have a subscript greater than $l$. Hence a finite number of reductions of the type (17) will suffice to bring $\left(A_{j}, T_{i}\right)$ into the form $A_{1} \gamma_{j i 1} \cdots A_{r} \gamma_{j i r}$.

We have outlined a method for constructing, from the data of an $\omega$-normal $U$-basis, a representation of $G$ by a subgroup of the group $G L_{p}\left(\delta_{1}, \delta_{2}, \cdots, \delta_{r}\right)$ of $r$-rowed $l$-matrices. Presently we shall extend this $p^{\gamma}-1$ representation of $G$ to a 1-1 representation by imbedding each matrix $E_{r}+M_{i}$ in an $(r+1)$ -

* The proof of this assertion is similar to the proof of Theorem I in $\$ 2$. In interchanging the $P$ 's we make use of the formula $P_{\beta}^{y} P_{\alpha}^{x}=P_{\alpha}^{x} P_{\beta}^{y}\left(P_{\beta}, P_{\alpha}\right)^{x y}, \beta>\alpha$, which can be derived from (16); a basis for induction is provided by the fact that the exponent of each constituent $P_{\lambda}$ in the normal form of $\left(P_{\beta}, P_{\alpha}\right)$ is divisible by $p$ for $\lambda \leqq \beta$. 
rowed $l$-matrix. First, however, we list several interesting properties of the matrices $M_{i}$ :

(18) The highest power of $p$ which divides $\gamma_{j i k}$ divides $\alpha_{j i k}$, and conversely.

(19) Every element in and below the main diagonal of each $M_{i}$ is divisible by $p$.

(20) For $j>k, \gamma_{j i k}$ is divisible by $p^{\delta_{k}-\delta_{j}}$.

The truth of (18) follows from the details of (17) and from the fact that this reduction is reversible; i.e., we may reduce $\left(A_{1}^{\gamma_{j i 1}} \cdots A_{r}^{\gamma_{j i r}}\right)^{-a-1}$ to the form $P_{1} \alpha_{j i 1} \ldots P_{r} \alpha_{j i r}$ if we replace $A_{k}$ by $T_{k}^{-1} P_{k}$. Obviously (19) and (20) follow directly from (18), or we may derive (20) from the fact that the order of $\left(A_{k}, T_{i}\right)$ must divide the order of $A_{k}$.

Since $\left(A_{i}, T_{j}\right)=\left(A_{i}, T_{i}\right)^{-1}$, we have the relation

$$
\gamma_{i j k}=p^{\delta_{k}}-\gamma_{i j k} \text {. }
$$

For $i=j$, this gives

$$
\gamma_{j j k}=0,
$$

where this zero is the residue 0 modulo $p^{\delta_{k}}$.

Let $M_{0}$ denote the $r$-rowed $l$-matrix in which each element in the $j$ th column is the residue 0 modulo $p^{\delta_{i}}, j=1,2, \cdots, r$. Let $R_{l i}$ denote the $r$-rowed $l$-matrix whose $l$ th row is $\gamma_{l i 1}, \gamma_{l i 2}, \cdots, \gamma_{l i r}$ and whose remaining rows contain only zeros. Now the least positive residues of the $l$ th row in the product $R_{l i} M_{j}$ are the exponents of the commutator $\left(\left(A_{l}, T_{i}\right), T_{j}\right)$ (written, of course, in the normal form $\left.A_{1}^{\lambda_{1}} A_{2}{ }^{\lambda_{2}} \cdots A_{r}^{\lambda_{r}}\right)$. Since this commutator is the identity of $G_{a}$, we see that $R_{l i} M_{j}$ must equal $M_{0}$. But $M_{i}=R_{1 i}+R_{2 i}+\cdots+R_{r i}{ }^{*}$ This establishes an important property of the matrices $M_{i}$, namely,

$$
M_{i} M_{j} \equiv M_{0}, \quad i, j=1,2, \cdots, r \text {. }
$$

For $i=j$, this gives

$$
M_{i}^{2} \equiv M_{0} .
$$

Let $H_{M}$ denote the group generated by the matrices $E_{r}+M_{i}, i=1,2, \cdots, r$. As we have seen above, $H_{M}$ is a 1-1 representation of $H$ and a $p^{\gamma-1}$ representation of $G$. The element of $H_{M}$ which corresponds to the "general" element $P_{1}^{x_{1}} P_{2} x_{2} \ldots P_{r}^{x_{r}}$ of $G$ is the $l$-matrix derived from $\left(E_{r}+M_{1}\right)^{x_{1}}\left(E_{r}+M_{2}\right)^{x_{2}} \ldots$

* See note on $l$-matrices in $\$ 3$. The matrices $M_{i}, R_{j i}$ are elements of a ring.

$\dagger$ We wish to emphasize the fact that $M_{i} M_{j}$ is to be regarded not as the product of $M_{i}$ and $M_{j}$ in the ordinary sense, but as the $l$-matrix defined by this product. 
$\left(E_{r}+M_{r}\right)^{x_{r}}$; and from (23) we see that this product can be represented in the simple form*

$$
\left(E_{r}+M_{1}\right)^{x_{1}} \cdots\left(E_{r}+M_{r}\right)^{x_{r}} \equiv E_{r}+\sum_{k=1}^{r} x_{k} M_{k} .
$$

We shall now construct a 1-1 representation of $G$ as a subgroup of the group $G L_{p}\left(\delta_{0}, \delta_{1}, \delta_{2}, \cdots, \delta_{r}\right)$, where $\delta_{0}$ is any fixed integer not less than $\delta_{1}$. First, we define $M_{i}^{\prime}$ as the $(r+1)$-rowed $l$-matrix

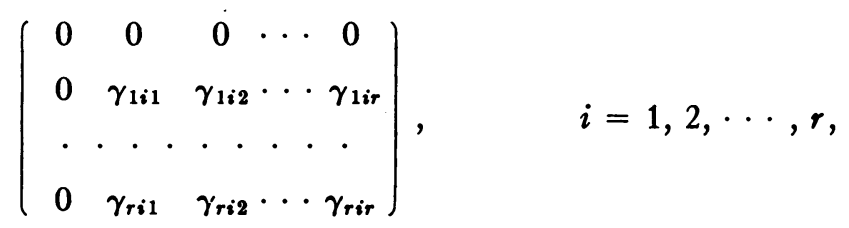

where the elements in the first column are the residues 0 modulo $p^{\delta_{0}}$. In order to avoid altering much of our earlier notation, we shall number the rows (and columns) in $M_{i}^{\prime}$ (and in the other $(r+1)$-rowed matrices which we shall presently define) by the sequence $0,1,2, \cdots, r$. Let $E^{\prime}$ be the identity matrix $\dagger$ of $G L_{p}\left(\delta_{0}, \delta_{1}, \cdots, \delta_{r}\right)$. It is at once evident that the matrices $E^{\prime}+M_{i}^{\prime}$ generate a group $G_{M}$ which is simply isomorphic with $H_{M}$.

We denote by $L_{i}^{\prime}$ the $(r+1)$-rowed $l$-matrix which has in row 0 and column $i$ the residue 1 modulo $p^{\delta_{i}}$ and zeros elsewhere. We shall denote the sum $M_{i}^{\prime}+L_{i}^{\prime}$ by the symbol $N !$.

The main result of this section is the following:

THEOREM I. The $r$ matrices $E^{\prime}+N_{i}^{\prime}$ generate a group $G_{N}$ which is simply isomorphic with $G$ under the correspondence defined by $P_{i} \sim E^{\prime}+N_{i}^{\prime}, i=1,2, \cdots, r$.

In proving this theorem we shall make use of the following known result: If $B_{1}, B_{2}, \cdots, B_{r}$, of orders $p^{\delta_{1}}, p^{\delta_{2}}, \cdots, p^{\delta_{r}}$ respectively, constitute a $U$ basis for an abelian group of order $p^{m}$, and if $\Theta_{1}, \Theta_{2}, \cdots, \Theta_{r}$ are a set of automorphisms of this group, each being defined by the $r$ equations

$$
\left(B_{i}, \Theta_{i}\right)=B_{1}^{\gamma_{j i 1}} \cdots B_{r}^{\gamma_{j i r}},
$$

where the $\delta$ 's and the $\gamma_{j i k}$ are the same as in (9) and (15) above, then the $p^{m}$ products $\Theta_{i} B_{i}, i=1,2, \cdots, r$, generate a group which is simply isomorphic with $G$ under the correspondence $P_{i} \sim \Theta_{i} B_{i} . \ddagger$

* That is, both sides of this equation define the same $l$-matrix of $H_{M}$.

$\dagger$ At this point we shall drop the subscripts from the identity matrices.

$\ddagger$ This result is contained implicitly in the paper on metabelian groups which has been quoted above (see $H, p .193)$. It is proved there that the $p^{m}$ products $\Theta_{1}^{x_{1}} \cdots \Theta_{r}^{x_{r}} B_{1}^{x_{1}} \cdots B_{r}^{x_{r}, 0 \leqq} x_{i}<p^{\delta_{i}}$, constitute a group simply isomorphic with $G$; and it is easy to see that one may bring into this form any product $\left(\Theta_{\alpha} B_{\alpha}\right)^{y_{\alpha}} \cdots\left(\Theta_{\beta} B_{\beta}\right)^{\boldsymbol{y}} \beta \cdots$. 
To prove Theorem $I$ it is therefore sufficient to show that

(i) $E^{\prime}+N_{i}^{\prime}$ equals $U_{i} V_{i}$, where $U_{i}$ and $V_{i}$ denote $E^{\prime}+M_{i}^{\prime}$ and $E^{\prime}+L_{i}^{\prime}$, respectively;

(ii) $V_{1}, V_{2}, \cdots, V_{r}$ are of orders $p^{\delta_{1}}, p^{\delta_{2}}, \cdots, p^{\delta_{r}}$ respectively, generate an abelian group, and constitute a $U$-basis for this group;

(iii) the $U_{i}$ and $V_{j}$ satisfy the equations

$$
\left(V_{j}, U_{i}\right)=V_{1}^{\gamma_{j i 1}} \ldots V_{r}^{\gamma_{j i r}}, \quad i<j ; i=1, \cdots, r ; j=2, \cdots, r .
$$

First, we write several useful formulas, which can be verified from the rules for multiplying $l$-matrices:*

$$
\begin{array}{cc}
L_{i} L_{j} \equiv M_{0 j} M_{i} M_{j} \equiv M_{0} \quad((23) \text { above }) ; & M_{i} L_{j} \equiv M_{0} ; \\
L_{j} M_{i} \equiv \gamma_{j i 1} L_{1}+\cdots+\gamma_{j i r} L_{r}, & i, j=1,2, \cdots, r . \\
U_{i}^{x_{i}}=\left(E+M_{i}\right)^{x_{i}} \equiv E+x_{i} M_{i} ; V_{i}^{y_{j}}=\left(E+L_{j}\right)^{y_{j}} \equiv E+y_{j} L_{j} ; \\
U_{1}^{x_{1}} \cdots U_{r}^{x_{r}} \equiv E+\sum_{k=1}^{r} x_{k} M_{k} ; \quad V_{1}^{\nu_{1}} \cdots V_{r}^{\nu_{r}} \equiv E+\sum_{k=1}^{r} y_{k} L_{k} .
\end{array}
$$

To prove (i) we have $U_{i} V_{i}=\left(E+M_{i}\right)\left(E+L_{i}\right) \equiv E+M_{i}+L_{i}+M_{i} L_{i} \equiv E+M_{i}+L_{i}=E+N_{i}$. (By definition, $N_{i}=L_{i}+M_{i}$; from (26), $M_{i} L_{i} \equiv M_{0}$.)

We now prove (ii). The equation $V_{i}^{y_{i}}=E$ requires $E+y_{i} L_{i} \equiv E$. Since $p^{\delta_{i}}$ is the smallest value of $y_{i}$ for which $y_{i} L_{i} \equiv M_{0}$, it follows that the order of $V_{i}$ is exactly $p^{\delta_{i}}$. The permutability of $V_{i}$ and $V_{j}$ follows from (26). For $V_{1}, V_{2}, \cdots, V_{r}$ to constitute a $U$-basis for the abelian group which they generate, it is sufficient that the equation $V_{1}^{y_{1}} \cdots V_{r}^{y_{r}}=E$ be satisfied only by $y_{i} \equiv 0 \bmod p^{\delta_{i}}$. That this is the case follows directly from (27) and the linear independence of the $L$ 's.

Finally we prove (iii). From (26) and (27) we derive the equalities $\dagger$

$$
\begin{aligned}
\left(V_{i}, U_{i}\right)=V_{i}^{-1} U_{i}^{-1} V_{j} U_{i} & \equiv\left(E-L_{j}\right)\left(E-M_{i}\right)\left(E+L_{j}\right)\left(E+M_{i}\right) \\
& \equiv\left(E-L_{j}-M_{i}+L_{j} M_{i}\right)\left(E+L_{j}+M_{i}+L_{j} M_{i}\right) \\
& \equiv E+\sum_{k=1}^{r} \gamma_{j i k} L_{k} \\
& \equiv \prod_{k=1}^{r}\left(E+L_{k}\right)^{\gamma_{j i k}}=V_{1}^{\gamma_{j i 1}} V_{2}^{\gamma_{j i 2}} \cdots V_{r}^{\gamma_{j i r}} .
\end{aligned}
$$

* We shall drop all primes, since from this point on we shall deal exclusively with $(r+1)$-rowed matrices. Note that $M_{0}$ is now the $(r+1)$-rowed null-matrix of the set $L_{p}\left(\delta_{0}, \delta_{1}, \cdots, \delta_{r}\right)$.

$\dagger$ It is understood, of course, that the notation $E-L_{j}$ is merely a convenient substitute for $E+\left(p^{\delta_{i}}-1\right) L_{j}$. 
This completes the determination of our 1-1 representation of $G$ by means of $l$-matrices. As we have seen, this particular representation $G_{N}$ is completely defined by the orders of the basis elements $P_{1}, P_{2}, \cdots, P_{r}$ of $G$, the exponents $\alpha_{j i k}$ in the $r(r-1) / 2$ equations (10), and the permutability relation $\left(P_{i}, P_{j}\right) P_{k}=P_{k}\left(P_{i}, P_{j}\right)$. In respect to the totality of possible representations of $G$ by $l$-matrices $L_{p}\left(\delta_{1}, \delta_{2}, \cdots, \delta_{r}\right)$, the representation $G_{N}$ may be regarded as a normal form, in that for every matrix in $G_{N}$ the elements in and below the main diagonal are congruent modulo $p$ to 1 and 0 respectively.

We observe that the matrices $E+\sum_{k=1}^{r} x_{k} L_{k}, 0 \leqq x_{k}<p^{\delta_{k}}$, define a 1-1 representation of the abelian group $G_{a}$, and the corresponding elements of $G_{N}$ are the $l$-matrices derived from the products $\left(E+\sum_{k=1}^{r} x_{k} M_{k}\right)\left(E+\sum_{k=1}^{r} x_{k} L_{k}\right)$ $\equiv E+\sum_{k=1}^{r} x_{k} N_{k}$ (see (8) and (25) above). Thus we obtain all the elements of $G_{N}$ from a "general" matrix, whose coordinates are linear functions of $r$ parameters, by specializing these parameters and taking least positive residues. We shall see, later, that the $N$ 's combine under multiplication according to the linear formula $N_{i} N_{j} \equiv \sum_{k=1}^{r} \gamma_{i j k} N_{k}$.

5. The notation which we shall use in this section is that of the preceding. Our objective is to characterize those matrices of the group $G L_{p}\left(\delta_{1}, \delta_{2}, \cdots, \delta_{r}\right)$ which represent automorphisms* of $G$. We know, of course, that the group of isomorphisms of $G_{a}$ is simply isomorphic with $G L_{p}\left(\delta_{1}, \delta_{2}, \cdots, \delta_{r}\right)$; and from (7) of $\$ 4$ it follows that $I(G)$ is simply isomorphic with a certain subgroup of $G L_{p}\left(\delta_{1}, \cdots, \delta_{r}\right)$. Now every matrix $X=\left(x_{i j}\right)$ in $G L_{p}\left(\delta_{1}, \cdots, \delta_{r}\right)$ is characterized by two conditions on the coordinates $x_{i j}$, namely,

$$
\begin{aligned}
x_{i j} & \equiv 0 \bmod p^{\delta_{i}-\delta_{i}} \text { for } i>j ; \\
\left|x_{i j}\right| & \neq 0 \bmod p .
\end{aligned}
$$

Our problem, therefore, is to determine the additional restrictions which must be imposed on the coordinates $x_{i j}$ in order that $x$ shall define an automorphism of $G$. It is possible to determine these additional conditions as congruences involving the $\alpha_{j i k}$ by regarding $X$ as the coefficient-matrix in the correspondence

$$
\left\{\begin{array}{c}
P_{1} \sim P_{1}^{x_{11}} \cdots P_{r}^{x_{1 r}} \\
\cdot \cdot \cdot \cdot \cdot \\
P_{r} \sim P_{1}^{x_{r 1}} \cdots P_{r}^{x_{r r}} .
\end{array}\right.
$$

It is much easier, however, to determine them in terms of the $\gamma_{j i k}$, and this is

* Throughout this article the term "automorphism" of $G$ denotes a 1-1 isomorphism of $G$ with itself. 
the procedure which we shall adopt. First, we regard $X$ as the coefficientmatrix of the "general" automorphism of $G_{a}$, defined by the correspondence

$$
\left\{\begin{array}{c}
A_{1} \sim B_{1}=A_{1}^{x_{11}} \cdots A_{r}^{x_{1 r}} \\
\cdots \cdot \cdots \cdot \cdots \cdot \\
A_{r} \sim B_{r}=A_{1}^{x_{r 1}} \cdots A_{r}^{x_{r r}}
\end{array}\right.
$$

the $x_{i j}$ being least positive residues.

Let $\pi_{x}$ denote that permutation of $I\left(G_{a}\right)$ which is defined by $X$. Now $\pi_{x}$ transforms $G$ either into itself or a conjugate permutation group in $K\left(G_{a}\right)$; furthermore, $G$ is generated by the $r$ permutations $P_{i}=T_{i}, i=1, \cdots, r$ (see (11) and (12) of $\$ 4$ ). Hence, for $X$ to define an automorphism of $G$ it is necessary and sufficient that $\pi_{x}^{-1} T_{i} A_{i} \pi_{x}, x=1,2, \cdots, r$, be a permutation of $G$. Now the permutation of $G$ which corresponds to the permutation $A_{z}=A_{1}{ }_{1} \cdots A_{r}{ }^{z}$ of $G_{a}$ is the product $T_{z} A_{z}$, where $T_{z}$ is $T_{1}^{z_{1}} \cdots T_{r}{ }^{z_{r}}$ (see (8) of $\$ 4$ ). Consequently, for $X$ to define an automorphism of $G$ it is necessary and sufficient that

$$
\pi_{x}^{-1} T_{i} \pi_{x} \text { be equal to } T_{1}^{x_{i 1}} \cdots T_{r}^{x_{i r}}, \quad i=1,2, \cdots, r .
$$

The sufficiency of this condition is obvious. That it is necessary, as well, follows from the fact that if we multiply a given permutation $A_{z}=A_{1}{ }^{z_{1}} \cdots A_{r}{ }_{r}$, $0 \leqq z_{i}<p^{\delta_{i}}$, on the left by all the permutations of $H$, then only one of these products will be a permutation in $G$; that is, the multiplying permutation $T_{\mathrm{s}}$ is unique, although its representation in the form $T_{1^{z_{1}}} \cdots T_{r_{r}}^{z_{r}}, 0 \leqq z_{i}<p^{\delta_{i}}$, is never unique.

We seek a more explicit form for condition (5). As in the preceding section, each $T_{i}$ is equivalent to a substitution

$$
\left\{\begin{array}{c}
A_{1} \rightarrow A_{1}^{\prime}=A_{1}^{1+\gamma_{1 i 1}} \cdots A_{r}^{\gamma_{1 i r}} \\
\cdot \cdot \cdot \cdot \cdot \cdot \cdot \cdot \cdot \\
A_{r} \rightarrow A_{r}^{\prime}=A_{1}^{\gamma_{r i 1}} \cdots A_{r}^{\gamma_{r i r}},
\end{array}\right.
$$

whose matrix we have already denoted by $E+M_{i}$. Similarly, the product $T_{1}^{x_{i 1}} \cdots T_{r}{ }^{x_{i r}}$ is equivalent to a substitution on the $A$ 's whose matrix is given by $E+\sum_{k=1}^{r} x_{i k} M_{k}$ (see (25) of $\$ 4$ ). And the matrix of that substitution (on the $A$ 's) which is equivalent to the permutation $\pi_{x}^{-1} T_{i} \pi_{x}$ is clearly equal to $X^{-1}\left(E+M_{i}\right) X$. Hence (5) is equivalent to the equations

$$
E+\sum_{k=1}^{r} x_{i k} M_{k} \equiv X^{-1}\left(E+M_{i}\right) X, \quad i=1, \cdots, r ;
$$


and these, in turn, are equivalent to the set

$$
\sum_{k=1}^{r} x_{i k} M_{k} \equiv X^{-1} M_{i} X
$$

The main result of this section may be expressed by the theorem:

The group of isomorphisms of $G$ is simply isomorphic with the group generated by those l-matrices $L_{p}\left(\delta_{1}, \cdots, \delta_{r}\right)$ for which the following conditions are satisfied:

$$
\begin{array}{rlrl}
x_{i j} & \equiv 0 \bmod p^{\delta_{i}-\delta_{i}} \quad \text { for } \quad i>j ; \\
\left|x_{i j}\right| & \neq 0 \bmod p ; & & \\
\sum_{k=1}^{r} x_{i k} M_{k} & \equiv X^{-1} M_{i} X, & & i=1,2, \cdots, r .
\end{array}
$$

In conclusion, we state a useful relation, namely,

$$
\sum_{k=1}^{r} \gamma_{i j k} M_{k} \equiv M_{0}, \quad i=1,2, \cdots, r_{2}
$$

which can be derived by substituting $E+M_{i}$ for $X$ in (7) above.

6 . In this section we shall investigate the abstract structure of the representation $G_{N}$, and we shall see that the matrices $E, N_{1}, \cdots, N_{r}$ may be regarded as basis-units in a certain finite ring.

We have already pointed out that the general element of $G_{N}$ is obtained by reducing the matrix $J_{x}=E+\sum_{k=1}^{r} x_{k} N_{k}$, where each $x_{k}$ ranges from 0 to $p^{\delta_{k}}-1$. Since the product $J_{z}=J_{x} J_{y}$ must occur in the form $J_{z}=E+\sum z_{k} N_{k}$, each of the $r^{2}$ products $N_{i} N_{j}$ must obviously be equivalent to a linear function of the matrices $E, N_{1}, \cdots, N_{r}$. This linear relation is given by the formula

$$
N_{i} N_{j} \equiv \sum_{k=1}^{r} \gamma_{i j k} N_{k} .
$$

In deriving this formula we replace $N_{i}$ by $L_{i}+M_{i}{ }^{*}$ From (26) of $\$ 4$ and (8) of $\$ 5$ we have the chain of equations

$$
\begin{aligned}
N_{i} N_{i}=\left(L_{i}+M_{i}\right)\left(L_{j}+M_{j}\right) \equiv L_{i} M_{j} & \equiv \sum_{k=1}^{r} \gamma_{i j k} L_{k} \equiv \sum_{k} \gamma_{i j k} L_{k}+\sum_{k} \gamma_{i j k} M_{k} \\
& \equiv \sum_{k} \gamma_{i j k}\left(L_{k}+M_{k}\right) \equiv \sum_{k=1}^{r} \gamma_{i j k} N_{k} .
\end{aligned}
$$

* Observe that $M_{i}$ is the $(r+1)$-rowed matrix $M_{i}^{\prime}$ of $\$ 4$. It is evident that (8) above is valid if we replace $M_{i}$ by $M_{i}^{\prime}$. 
Since $\gamma_{i i k} \equiv-\gamma_{i j k} \bmod p^{\delta_{k}}$ and $\gamma_{i i k} \equiv 0 \bmod p^{\delta_{k}}($ see (21) and (22) of $\S 4)$, we have for the $N$ 's the further relations

$$
N_{i} N_{j} \equiv-N_{j} N_{i}
$$

(the interpretation of this congruence is obvious);

$$
N_{i}^{2} \equiv N_{0}
$$

where $N_{0}$ is the $(r+1)$-rowed null matrix.

From (1) and (2) it is easy to show that the product of any three of the $N$ 's is the null matrix; that is,

$$
N_{i} N_{j} N_{k} \equiv N_{0}, \quad i, j, k=1,2, \cdots, r .
$$

At this point it is fairly evident that with the group $G_{N}$ there is associated a finite ring having as basis-units the matrices $E, N_{1}, \cdots, N_{r}$. We wish to show that this ring can be constructed without assuming the existence of $G_{N}$.

We start with a system $\delta$ of double composition in which all the ring postulates are satisfied except (possibly) associativity of multiplication. We designate a set of $r+1$ linearly independent basis-units for $\delta$ by $v_{e}, v_{1}, \cdots, v_{r}$, and we assume that every element of $\delta$ can be represented uniquely in the form $v_{x}=x_{0} v_{e}+\sum_{i=1}^{r} x_{i} v_{i}$, where the $x_{i}$ are arbitrary rational integers.

We assume that multiplication for the basis-units (and accordingly for every element of $S$ ) is defined by the equations

$$
\left\{\begin{array}{rl}
v_{e}^{2} & =v_{e}, \\
v_{e} v_{i} & =v_{i} v_{\bullet}=v_{i}, \\
v_{i} v_{j} & =\sum_{k=1}^{r} \gamma_{i j k} v_{k}, *
\end{array} \quad i=1,2, \cdots, r\right.
$$

where the $\gamma_{i j k}$ have the same values as in (1) above. We recall that each $\gamma_{i j k}$ is a positive integer less than $p^{\delta_{k}}$, and that these $r^{3}$ integers satisfy

$$
\begin{aligned}
& \gamma_{i j k} \equiv-\gamma_{j i k} \bmod p^{\delta_{k}} \quad[\S 4,(21)] \\
& \left.\gamma_{i j k} \equiv 0 \quad \bmod p^{\delta_{k}-\delta_{i}} \text { for } i>k\right\} \\
& \left.\gamma_{i j k} \equiv 0 \quad \bmod p^{\delta_{k}-\delta_{j}} \text { for } j>k\right\} \\
& \sum_{\alpha=1}^{r} \gamma_{i j \alpha} \gamma_{\alpha k l} \equiv 0 \quad \bmod p^{\delta_{l}} \\
& \sum_{\alpha=1}^{r} \gamma_{j k \alpha} \gamma_{i \alpha l} \equiv 0 \quad \bmod p^{\delta_{l}} \\
& {[\$ 4,(20) \text { and }(21)]} \\
& {[\S 5,(8)] \text {. }}
\end{aligned}
$$

* It is known, of course, that the various assumptions above are always consistent. In fact, a system of the type $S$ exists if we replace the $\gamma_{i j k}$ in (5) by $r^{3}$ arbitrary integers; and if multiplication is associative (which is generally not the case), then this system is a ring of rank $r+1$, having the ring of integers as its coefficient-domain. 
Now those elements $v_{x}$ for which $x_{k}$ is divisible by $p^{\delta_{k}}, k=0,1, \cdots, r{ }^{*}$ constitute under addition a modulus $\mathcal{X}$. We wish to show that the elements of this modulus constitute an invariant ideal in $\mathcal{S}$. Since they form a modulus, it is sufficient to show that $v_{x} \alpha_{y}$ and $\alpha_{y} v_{x}$ are each of the form $\lambda_{0} p^{\delta_{0} v_{e}}+\sum_{i=1}^{r} \lambda_{i} p^{\delta_{i} v_{i}}$, where $v_{x}$ and $\alpha_{y}$ are any elements of $\delta$ and $\mathcal{X}$ respectively.

From (5) we have $\dagger$

$$
\left(\sum_{i=1}^{r} x_{i} v_{i}\right)\left(\sum_{j=1}^{r} y_{j} p^{\delta} i v_{j}\right)=\sum_{k=1}^{r}\left[\sum_{i=1}^{r} x_{i}\left(\sum_{j=1}^{r} y_{i} p^{\delta} \gamma_{i j k}\right)\right] v_{k} .
$$

Since $\delta_{1} \geqq \delta_{2} \geqq \cdots \geqq \delta_{r}$, it is obvious that when $k$ is at least equal to both $i$ and $j$, then the coefficient of $v_{k}$ is divisible by $p^{\delta_{k}}$. From (7), however, we know that $\gamma_{i j k}$ has the form $\gamma_{i j k}^{\prime} p^{\delta_{k}-\delta_{i}}$ (or $\gamma_{i j k}^{\prime \prime} p^{\delta_{k}-\delta_{i}}$ ) for $k<i$ (for $k<j$ ). In every case then, the coefficient of $v_{k}$ is divisible by $p^{\delta_{k}}, k=0,1, \cdots, r$. Similarly, we may show that $\alpha_{y} v_{x}$ is an element of $\mathcal{X}$. We denote this invariant ideal by the letter $\Im$.

From a familiar result in the theory of ideals we know that the residue classes of $\mathcal{S}$ with respect to $J$ form a system $\bar{\delta}$ which is homomorphic with $\delta$. If we denote by $u_{e}, u_{1}, \cdots, u_{r}$ the elements of $\bar{S}$ to which $v_{e}, v_{1}, \cdots, v_{r}$ correspond respectively in this homomorphism, then it is easy to see that the $u$ 's constitute a basis for $\overline{\mathcal{S}}$, and that every element of $\overline{\mathcal{S}}$ can be represented in the form $u_{x}=\left[x_{0}\right] u_{e}+\sum_{i=1}^{r}\left[x_{i}\right] u_{i}$, where $\left[x_{i}\right]$ is the symbol for the class of integers congruent to $x_{i}$ modulo $p^{\delta_{i}}$. In view of the homomorphism above, we know that the sum $u_{x}+u_{y}$ is represented by $\left[x_{0}+y_{0}\right] u_{0}+\sum_{i}\left[x_{i}+y_{i}\right] u_{i}$, while the multiplication table for the $u$ 's is given by

$$
\begin{aligned}
u_{e}^{2} & =u_{e}, \\
u_{e} u_{i} & =u_{i} u_{e}=u_{i}, \\
u_{i} u_{i} & =\sum_{k=1}^{r} \gamma_{i j k} u_{k} .
\end{aligned} \quad i=1,2, \cdots, r
$$

The condition for associativity of multiplication in $\bar{\S}$ is given by

$$
\left[\sum_{\alpha=1}^{r}\left[\gamma_{i j \alpha}\right]_{\alpha}\left[\gamma_{\alpha k l}\right]_{l}\right]_{l}=\left[\sum_{\alpha=1}^{r}\left[\gamma_{j k \alpha}\right]_{\alpha}\left[\gamma_{i \alpha l}\right]_{l}\right]_{l} . \ddagger
$$

But from (8) and (9) we know that both sides of this equation are equal to the residue class 0 modulo $p^{\delta_{l}}$. Hence $\bar{\delta}$ is a ring, since it is homomorphic

* These $p^{\delta_{k}}$ are the type-invariants of $G$ [see $\left.\$ 4\right]$.

$\dagger$ Obviously $v_{x} \cdot y_{0} \phi^{\delta_{0}} v_{e}$ is in $\mathcal{X}$.

$\ddagger$ The symbol $[\xi]_{\lambda}$ denotes the class of integers having the form $\xi \pm n p^{\delta \lambda}$. We need consider only elements in $\bar{S}$ of the form $\sum_{i=1}^{r}\left[x_{i}\right] u_{i}$, since $\left(u_{e} u_{x}\right) u_{y}$ is obviously equal to $u_{e}\left(u_{x} u_{y}\right)$. 
with a system $\delta$ which satisfies every ring postulate except associativity of multiplication.

From (6), which implies that $\left[\gamma_{i j k}\right]_{k}=\left[-\gamma_{j i k}\right]_{k}$, we may easily show that those elements in $\bar{\delta}$ which have the form $u_{x}{ }^{\prime}=\sum_{i=1}^{r}\left[x_{i}\right] u_{i}$ constitute a nilpotent* ring $R$ of index 3 in which the square of every element is the zero element. It is clear that $R$ is an invariant subring of $\bar{\delta}$; furthermore, the elements of $R$ constitute under addition an abelian group $A_{R}$ which is simply isomorphic with $G_{a}$ of $\$ 4$. One may also show that the elements of $\bar{\delta}$ having the form $u_{x}^{\prime \prime}=[1] u_{e}+u_{x}^{\prime}$ constitute under multiplication a group $G_{R}$ of order $p^{m}$ and class 2 . That they form a group is obvious, since $u_{x}^{\prime \prime} u_{y}^{\prime \prime}$ can be brought into the form [1] $u_{e}+u_{z}^{\prime}$, while every $u_{x}^{\prime \prime}$ has an inverse, namely, [1] $u_{e}+\sum_{i=1}^{r}\left[-x_{i}\right] u_{i}$. The commutator $\left(u_{i}^{\prime \prime}, u_{j}^{\prime \prime}\right)$, where $u_{i}^{\prime \prime}=u_{e}+u_{i}$, is $u_{e}+\sum_{\alpha=1}^{r} 2 \gamma_{i j \alpha} u_{\alpha}$, and by using (8) and (9) one readily computes for $\left(u_{i}^{\prime \prime}, u_{j}^{\prime \prime}\right) u_{k}^{\prime \prime}$ and $u_{k}^{\prime \prime}\left(u_{i}^{\prime \prime}, u_{j}^{\prime \prime}\right)$ the common value $u_{e}+u_{k}+\sum_{\alpha=1}^{r} 2 \gamma_{i j \alpha} u_{\alpha}$. Since $G_{R}$ is generated by the $u_{k}^{\prime \prime \prime}$ 's, its commutator subgroup must be contained in its central; hence $G_{R}$ is of class 2 .

Since $\bar{\delta}$ contains a principal unit $u_{e}$, we can construct a $1-1$ matrix-representation of $\bar{S}$ by the familiar process of post-multiplication. We denote the products $u_{e}\left(u_{e}+u_{i}\right), u_{1}\left(u_{e}+u_{i}\right), \cdots, u_{r}\left(u_{e}+u_{i}\right)$ by $\bar{u}_{e}, \bar{u}_{1}, \cdots, \bar{u}_{r}$ respectively. Then the matrix-representation of the element $u_{e}+u_{i}$ is the coefficientmatrix of the linear transformation

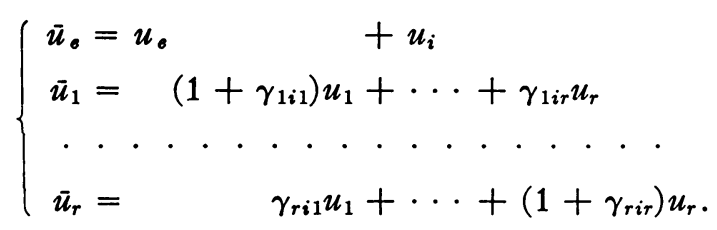

And this matrix is exactly the $l$-matrix $E+N_{i}$ of $G_{N}$. Since $G_{R}$ is generated by the $r$ elements $u_{e}+u_{i}, i=1,2, \cdots, r$, it follows that the group $G_{R}()$ is simply isomorphic with $G_{N}$, and consequently with $G$ (of $\$ 4$ ).

This representation of $G$ as a multiplicative group of elements in a finite ring which are congruent to the principal unit modulo a nil ring has conspicuous advantages for the study of the structure of $G$.† For instance, if $R^{\prime}$ is an invariant subring of $R$, then the elements of $G_{R}$ which have the form

* A nilpotent ring of index $k$ is a ring in which the product of any $k$ elements is 0 ; a nil ring is one in which some power of every element is 0 . A nil ring containing a finite basis is necessarily nilpotent.

$\dagger$ This method of "Strahlbildung" has been used to advantage by K. Shoda (Mathematische Annalen, vol. 100 (1928), pp. 674-686) for determining two important series of characteristic subgroups of $G L_{p}\left(\delta_{1}, \delta_{2}, \cdots, \delta_{r}\right)$. 
[1] $u_{e}+\rho, \rho$ in $R^{\prime}$, constitute an invariant subgroup of $G_{R}$. Thus the subgroup $\mho_{\alpha}\left(G_{R}\right)$ corresponds to the subring $\sum_{i=1}^{r}\left[x_{i} p^{\alpha}\right] u_{i}$ of $R$.

From the representation $G_{R}$ we can derive a second set of conditions on the coordinates of those matrices in $L_{p}\left(\delta_{1}, \delta_{2}, \cdots, \delta_{r}\right)$ which define automorphisms of $G$. Now any automorphism of the additive group in $R$ is defined by a linear transformation

$$
\Theta:\left\{\begin{array}{c}
u_{1}^{\prime}=y_{11} u_{1}+\cdots+y_{1 r} u_{r} \\
\cdots \cdot \cdots \\
u_{r}^{\prime}=y_{r 1} u_{1}+\cdots+y_{r r} u_{r}
\end{array}\right.
$$

in which the $y_{i j}$ are least positive residues satisfying the conditions

$$
\left|y_{i j}\right| \not \equiv 0 \bmod p ; y_{i j} \equiv 0 \bmod \phi^{\delta_{j}-\delta_{i}} \text { for } i>j .
$$

But $\Theta$ defines a correspondence of the elements of $G_{R}$, and for this correspondence to define an automorphism of $G_{R}$, it is clearly necessary and sufficient that the multiplication table of the $u_{i}^{\prime}$ 's be the same as that of the $u_{i}$ 's. By substituting the $u_{i}^{\prime}$ 's in equations (10) and taking account of the relations $u_{i}{ }^{2} \equiv 0$ and $u_{j} u_{i} \equiv-u_{i} u_{i}$ we find that $\Theta$ will define an automorphism of $G_{R}$ if, and only if, the $y$ 's satisfy the $r^{2}(r-1) / 2$ congruences

$$
\sum_{\mu, v} D_{i j-\mu v} \gamma_{\mu v k} \equiv \sum_{\alpha=1}^{r} \gamma_{i j \alpha} y_{\alpha k} \bmod p^{\delta_{k}},
$$

where $k=1, \cdots, r ; i<j ; i=1, \cdots, r ; j=2, \cdots, r ; \mu<v ; \mu=1, \cdots$, $r ; v=2, \cdots, r$, and where $D_{i j-\mu v}$ denotes the determinant

$$
\left|\begin{array}{ll}
y_{i \mu} & y_{i v} \\
y_{j \mu} & y_{j v}
\end{array}\right| .
$$

As one would expect, these congruences are equivalent to the matrixequations (7) in $\$ 5$.

The representation $G_{R}$ above was derived (although indirectly) from a particular $\omega$-normal $U$-basis of $G$, and our method is probably the simplest one for obtaining a ring-representation for $G$. But the existence of a $U$-basis is by no means necessary* for the existence of a representation of a finite $p$-group $\bar{G}$ as the multiplicative group of those elements in a ring which are congruent to the principal unit modulo a subring whose elements are expressible uniquely in the form $\sum_{i=1}^{i} \xi_{i} w_{i}$, the $\xi_{i}$ being least positive residues

* The quaternion group, which has no $U$-basis, is the multiplicative group of elements $\rho+\xi_{1} u_{1}$ $+\xi_{2} u_{2}+\xi_{3} u_{3}, i=0,1$, in the ring of 16 elements whose multiplication is defined by the equations $e^{2}=e$; $e u_{i}=u_{i} e=u_{i} ; u_{1}^{2}=u_{2}^{2}=u_{2} u_{1}=u_{3} ; u_{1} u_{3}=u_{3} u_{1}=u_{1} u_{2}=u_{2} u_{3}=u_{3} u_{2}=u_{3}^{2}=0$. 
modulo certain powers of $p$. Nor is it necessary, when $\bar{G}$ is the group $G$ above, that $\bar{r}$, the number of linearly independent basis units of $\bar{R}$, be equal to $r$, the number of type-invariants of $G$. For $\bar{r} \neq r$, however, the ring $\bar{R}$ must contain elements whose square is not zero, and although it is always nilpotent, it need not be of index 3 .

In conclusion, we point out that if the group $G$ is a direct product of groups $G^{\prime} \times G^{\prime \prime} \times \cdots$, then for each factor $G^{(i)}$ we can find an $\omega$-normal $U$-basis, and by the method given in $\$ \$ 4$ and 6 we can construct for each factor a ring-representation $G_{R^{(i)}}$. Then for $G$ itself we obtain a ring-representation if we replace $R$ in $\$ 6$ by the direct sum of the rings $R^{\prime}+R^{\prime \prime}+\cdots$. And from this representation we obtain, by post-multiplication, a representation of $G$ as the direct sum of matrix-representations $G_{N}{ }^{\prime}+G_{N}{ }^{\prime \prime}+\cdots$.

7. In the preceding section we proved that every metabelian group of prime-power order can be exhibited as a multiplicative group in a finite ring. And since every metabelian group is the direct product of its Sylow subgroups, one may construct for any metabelian group $\bar{G}$ of odd order a representation of this sort in which the ring $R$ is replaced by the direct sum of nil rings $R_{p_{1}}, R_{p_{2}}, \cdots$, each $R_{p_{\lambda}}$ corresponding to a Sylow subgroup of $\bar{G}$. We wish to show that there is, in a crude sense, a reciprocal relationship between nil rings and metabelian groups.

If $S$ is a ring which contains a principal unit e and a subring $\Sigma$ such that

(a) the square of every element in $\Sigma$ is the zero element, and

(b) the number of elements in $\Sigma$ is an odd integer $n$, then those elements in $S$ which are of the form $e+\sigma, \sigma$ in $\Sigma$, constitute under multiplication a group of order $n$ whose class does not exceed 2.

It is easy to show that the elements' $e+\sigma$ constitute under multiplication a group $F_{\Sigma}$ of order $n$, having $e$ as the identical operation. We therefore give only the proof that $F_{\Sigma}$ is either abelian or metabelian.

Let $\alpha$ and $\beta$ denote any two elements of $\Sigma$. From (a) we have

$$
\sigma^{2}=0 \text {, where } \sigma \text { is any element of } \Sigma \text {. }
$$

By substituting $\alpha+\beta$ for $\sigma$ in this equation, we obtain

$$
\alpha \beta+\beta \alpha=0 .
$$

Two cases arise:

Case A. $\alpha \beta=\beta \alpha$ for every pair of elements in $\Sigma$;

Case B. $\Sigma$ contains two elements $\sigma_{\alpha}$ and $\sigma_{\beta}$ such that $\sigma_{\alpha} \sigma_{\beta} \neq \sigma_{\beta} \sigma_{\alpha}$.

In Case A equation (2) reduces to $2 \alpha \beta=0$. Since $\Sigma$ contains a finite number of elements, with each element $\sigma_{i}$ there is associated a smallest positive 
integer $m_{i}$ such that $m_{i} \sigma_{i}=0$. Now the elements of $\Sigma$ constitute under addition an abelian group of order $n$, and since $m_{i}$ is clearly the order of $\sigma_{i}$ with respect to this group, we see that $m_{i}$, being a divisor of $n$, is necessarily odd. Hence the equation $2 \alpha \beta=0$ is possible only if $\alpha \beta=0$. In Case $\mathrm{A}$, therefore, any two elements $e+\alpha$ and $e+\beta$ are commutative, and $F_{\Sigma}$ is of class 1 .

For Case B we first prove that the product of any three elements of $\Sigma$ is zero. By using (2) and the associativity postulate, we obtain the equations

$$
\begin{aligned}
\left(\sigma_{\alpha} \sigma_{\beta}\right) \sigma_{\gamma} & =\sigma_{\alpha}\left(\sigma_{\beta} \sigma_{\gamma}\right)=-\left(\sigma_{\beta} \sigma_{\gamma}\right) \sigma_{\alpha}=-\sigma_{\beta}\left(\sigma_{\gamma} \sigma_{\alpha}\right)=\left(\sigma_{\gamma} \sigma_{\alpha}\right) \sigma_{\beta} \\
& =\sigma_{\gamma}\left(\sigma_{\alpha} \sigma_{\beta}\right)=-\left(\sigma_{\alpha} \sigma_{\beta}\right) \sigma_{\gamma} .
\end{aligned}
$$

That is, $2 \sigma_{\alpha} \sigma_{\beta} \sigma_{\gamma}=0$; and as in Case $\mathrm{A}$, we infer that $\sigma_{\alpha} \sigma_{\beta} \sigma_{\gamma}$ is zero. To prove that $F_{\Sigma}$ is of class 2 it is sufficient to show that the commutator $\left(e+\sigma_{\alpha}, e+\sigma_{\beta}\right)$ of any two elements in $F_{\Sigma}$ is commutative with any third element $e+\sigma_{\gamma}$. From (1) we find that the inverse of $e+\sigma_{\alpha}$ is $e-\sigma_{\alpha}$. By making use of (3), it is a simple matter to show that the commutator $\left(e+\sigma_{\alpha}, e+\sigma_{\beta}\right)$ is given by $e+\sigma_{\alpha} \sigma_{\beta}-\sigma_{\beta} \sigma_{\alpha}$ and is commutative with $e+\sigma_{\gamma}$.

Finally, we observe that the theorem above is valid if we replace (a) by the assumption that $\Sigma$ is nilpotent and of index 3 ; that is, the product of any three elements in $\Sigma$ is the zero-element. (In this case, the commutator $\left(e+\sigma_{\alpha}, e+\sigma_{\beta}\right)$ equals $e+\sigma_{\alpha} \sigma_{\beta}-\sigma_{\beta} \sigma_{\alpha}-\sigma_{\alpha}^{2}-\sigma_{\beta}{ }^{2}$.)

BROWN UNIVERSITY, PROVIDENCE, R. I. 